D.O.I.: $10.3895 / \mathrm{S} 1808-04482008000100006$

\title{
FLEXIBILIDADADE DO TRABALHO: UM ESTUDO SOBRE O BANCO DE HORAS EM UMA EMPRESA DE ELETRODOMÉSTICOS
}

\section{LABOR FLEXIBILITY: A CASE ABOUT ANNUAL JOURNEY CONTRACTS IN AN APPLIANCE ENTERPRISE}

\author{
Lílian Carneiro Souza ${ }^{1}$; Alessandra Rachid ${ }^{2}$ \\ ${ }^{1}$ Federal University of São Carlos - UFSCar - São Carlos - Brasil 1liancarneirosouza@yahoo.com.br \\ ${ }^{2}$ Federal University of São Carlos- UFSCar - São Carlos - Brasil arachid@power.ufscar.br
}

\begin{abstract}
Resumo
O objetivo deste trabalho é analisar as estratégias de flexibilidade do trabalho em uma empresa de eletrodomésticos de linha branca, em especial o banco de horas. A reorganização das empresas a partir da década de 1990 tem se conduzido pela busca de flexibilidade. No que diz respeito ao trabalho, as diferentes estratégias para obter a flexibilidade podem ser classificadas em dois tipos: flexibilidade funcional, que é a ampliação das funções exercidas pelos trabalhadores, e flexibilidade numérica, relacionada à redução do emprego por prazo indeterminado em tempo integral e ao crescimento de formas alternativas de contratação, na qual se enquadra o banco de horas. Para obter as informações sobre essas estratégias, foram realizadas entrevistas com gerentes da fábrica sobre seu processo de reestruturação. Sobre o banco de horas, em particular, a pesquisa envolveu entrevista com 51 trabalhadores, gerentes de recursos humanos e da fábrica supervisores da produção e um diretor do sindicato dos trabalhadores, para saber como avaliam essa forma de flexibilidade.
\end{abstract}

Palavras-chave: Organização do trabalho, flexibilidade do trabalho, jornada flexível.

\section{Introdução}

A reorganização das empresas a partir da década de 90 tem se conduzido pela busca de flexibilidade. No que diz respeito ao trabalho, as diferentes estratégias para obter a flexibilidade podem ser classificadas em dois tipos: flexibilidade funcional, que é a ampliação das funções exercidas pelos trabalhadores, e flexibilidade numérica, relacionada à redução do emprego por prazo indeterminado em tempo integral e ao crescimento de formas alternativas de contratação (Wood, 1989; Smith, 1997; Hirata, 1998).

O objetivo deste artigo é pesquisar as estratégias de flexibilidade, funcional e numérica, empregadas por uma empresa de eletrodomésticos de linha branca, em especial a flexibilidade numérica via banco de horas. 
Para obter as informações sobre essas estratégias, foram realizadas entrevistas com gerentes da fábrica sobre seu processo de reestruturação, que pode ser acompanhado por um período relativamente longo devido à execução de diferentes projetos que tiveram como projeto de pesquisa esta empresa. Como financiamento da FAPESP e do CNPq. Sobre o banco de horas, em particular, a pesquisa contou com uma bolsa de iniciação PIBIC-CNPq e mais um financiamento da FAPESP, envolvendo entrevista com 51 trabalhadores, gerentes de recursos humanos e da fábrica, supervisores da produção e um diretor do sindicato dos trabalhadores entre 2005 e 2006, para saber como avaliam essa forma de flexibilidade.

Este artigo está organizado da seguinte forma: o Item 2 apresenta brevemente a indústria de linha branca; o item 3, as diferentes formas de flexibilidade funcional e numérica, para no Item 4 aprofundar uma forma específica de flexibilidade numérica, conhecida no Brasil como Banco de Horas.

\section{O setor de linha branca}

A indústria de linha branca produz bens de consumo duráveis, eletrodomésticos não portáteis, como refrigeradores, lavadoras de roupas, freezers verticais e horizontais, lava - louças, secadoras de roupa, fogões, condicionadores de ar e fornos microondas (Rotta, 2004), que apresentam alta sazonalidade de consumo durante o ano. Este setor passou por um processo de transformação "impulsionado pelo avanço da tecnologia da informação" (Martinez, 2004, p. 18) e caracterizado pela:

- redução do número de empresas;

- internacionalização produtiva, com instalações em países de mercados emergentes como Leste Europeu, Sudeste Asiático e América Latina (Araújo et al, 2004 e Martinez, 2004);

- segmentação de mercado, em linhas completas de produtos para atender a mercados alvos (Invernizzi, 2000).

Segundo Araújo et al. (2004), o setor de linha branca pode ser classificado como um oligopólio misto mundial, isto é, oligopólio concentrado e diferenciado mundialmente, onde a competição e a produção se dão no âmbito mundial por um número reduzido de empresas e a característica central desta indústria é a diferenciação, seguida pela escala mínima eficiente. Estas características exigem que as empresas estejam constantemente renovando e inovando em produtos para que sejam rentáveis, no entanto, como o produto apresenta ciclo de vida curto, isto dificulta o crescimento do mercado ao longo do tempo.

Dois entraves presentes no setor de linha branca, a maturidade tecnológica, onde as inovações se dão em produtos e processos, e a elevada difusão dos produtos nos países 
desenvolvidos, que acarretava estagnação de mercado a partir da década de 90, levaram as empresas líderes a atitudes estratégicas, com os programas de reestruturação produtiva no âmbito internacional e a internacionalização produtiva, que levam as empresas a instalarem unidades em outras regiões, principalmente as em desenvolvimento e as integradas por blocos econômicos, como União Européia e MERCOSUL. Seus principais objetivos com esta estratégia são a ampliação do horizonte de rentabilidade, a busca de mercados internos e/ou regionais, a sustentação de mercados internos em países onde as empresas se instalam e dos países próximos, a exploração de novos mercados antes inexplorados, a redução dos custos de produção, de transação e de transporte, a adaptação dos produtos aos hábitos e gostos locais, aproximando-se mais de seus consumidores.

No Brasil, a desnacionalização da indústria de linha branca se deu entre as décadas de 1970 e 1990, que foi sendo incorporada por grandes multinacionais que se instalavam no Brasil, seja através de associações, controle de capital ou mesmo aquisições diretas (Araújo et al., 2004). As empresas que entraram no mercado brasileiro foram: Whirlpool (Multibrás), Electrolux, General Eletric (GE - Dako), Bosch/Siemens, LG, Springer Carrier, Esmaltec, Kärcher. A Semp Toshiba recentemente mostrou interesse em investir no setor (Rotta, 2004). O setor é um oligopólio com o mercado dominado por Whirlpool, EUA, e AB Electrolux, Suécia.

\section{A flexibilidade numérica e funcional do trabalho}

Existem diversas formas de utilizar a força de trabalho de maneira flexível. Atkinson (1986) apud Walby (2003) classifica as diferentes formas de flexibilidade relacionadas ao trabalho em dois tipos: funcional e numérica.

A flexibilidade funcional, qualitativa ou interna, relaciona-se a maior diversidade de tarefas e funções atribuídas aos trabalhadores (Atkinson, 1986 apud Walby, 2003; Hirata, 1998). Em várias atividades econômicas, tem havido uma diminuição do volume de emprego e um aumento da produtividade, devido à automação e ao processo de racionalização (Gadrey, 1999). Na indústria de linha branca, o processo de reestruturação gerou mudanças no volume do emprego e contribuiu para a elevação da produtividade. $\mathrm{Na}$ unidade pesquisada, o número de funcionários também vinha caindo. O Gráfico 1 apresenta essa evolução. Em 1980 haviam 3 mil empregados. No ano da pesquisa a fábrica estava com cerca de 1200 funcionários. Também tem havido uma redução do pessoal indireto, como engenheiros, gerentes e supervisores. Entre 1997 e 2002, a redução foi de $50 \%$, de 189 para 96 funcionários.

Gráfico 1 - Número de funcionários na unidade pesquisada 


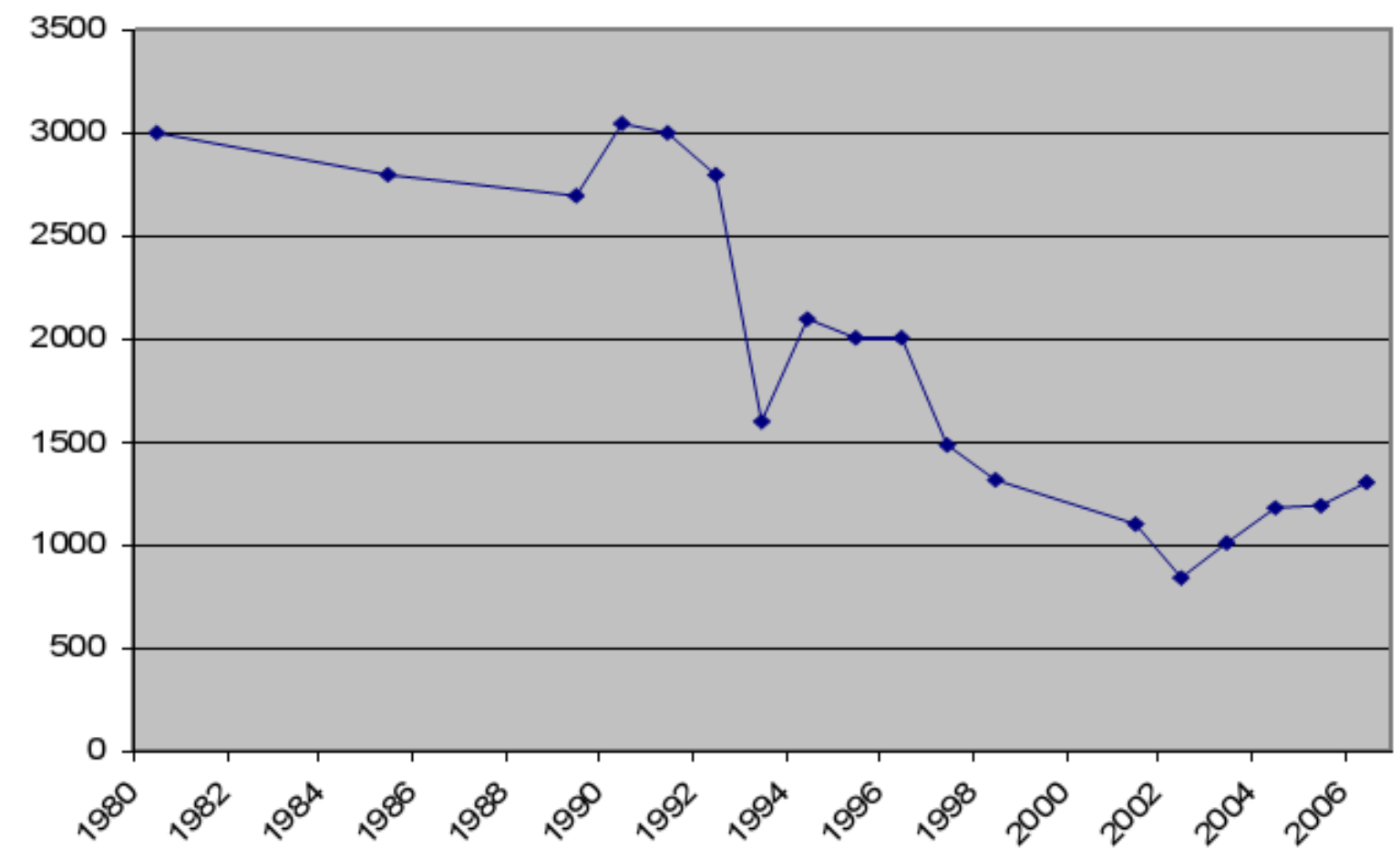

Devem ser numeradas em algarismos arábicos, apresentar título na parte superior e fonte na parte inferior, centralizado, fonte Times New Roman, tamanho 10, bem como ser referenciados no corpo do texto. Caso existam fotos ou figuras que demandem alta resolução, estas devem ser enviadas em arquivo separado em formato jpg e com a indicação da posição no texto.

As mudanças na organização da produção, a introdução de novos equipamentos, linhas de montagem mais flexíveis, novas ferramentas da qualidade e técnicas de planejamento e controle da produção têm gerado mudanças na divisão e no conteúdo do trabalho dentro da empresa, levando à ampliação das atribuições dos trabalhadores da produção, que passaram a transcender as tarefas diretamente relacionadas ao processo produtivo. A seguir, são apresentados alguns métodos de gestão da produção que têm levado à flexibilidade funcional:

- Automação: esta tem proporcionado, desde os anos 1990, a adoção de programas de manutenção preventiva de máquinas e equipamentos cuja maior preocupação, segundo um dos gerentes entrevistados, tornou-se a conscientização dos trabalhadores da produção no sentido do cuidado constante com os equipamentos por eles utilizados, o que envolve a lubrificação e a troca de óleo, a limpeza periódica das máquinas, a verificação de algum requisito que constitui parte de um programa de manutenção e a realização de inspeção das máquinas utilizadas.

- Just-in-time: Apesar de ser apontado com um dos principais métodos de gestão do modelo de produção enxuta, no Brasil, não tem sido muito utilizado. Na indústria de eletrodomésticos de linha branca seu uso é bastante restrito e os gerentes haviam 
desistido de implantá-lo na empresa pesquisada, continuando a atividade de programação da produção fortemente concentrada na área funcional específica, com pouca responsabilidade efetivamente transferida para os trabalhadores da produção.

- Qualidade: há a preocupação com a qualidade e a idéia de que os operários diretos devem ser envolvidos nas atividades de seu controle. Na empresa pesquisada, um dos gerentes entrevistados disse que os trabalhadores controlam a qualidade do que produzem, o que é auditado pela área da qualidade. Adicionalmente, tem-se um teste de 100\% no fim da produção. Na fábrica, utilizava-se um "diário de bordo" para controlar cada etapa da produção, que continha a quantidade produzida por hora, produtividade, tempo de parada, consumo de material, problemas de qualidade, etc. Cada operador ou facilitador digitava as informações do "diário de bordo" em um banco de dados e um grupo analisava os principais problemas de qualidade com o objetivo de gerar um plano de ação corretiva para melhoria dos processos com base no PDCA (Plan, Do, Check and Action).

- Grupos para solução de problemas: Na empresa pesquisada, os grupos de Kaisen entre os operários têm sido utilizado como forma de solução de problemas.

- Grupos semi-autônomos: Na indústria de linha branca, não foram observados avanços neste sentido. A maior parte dos operários se concentram nas linhas de montagem, bastante intensivas em trabalho, e devem executar tarefas dentro dos limites de tempo pré-estabelecidos, em ciclos curtos que se repetem inúmeras vezes ao longo da jornada de trabalho. Apesar disso, perguntados sobre a existência de trabalho em grupo, quase todos os entrevistados disseram estar familiarizados ao termo, mas referindo-se à colaboração e ajuda mútua e a grupos de solução de problemas. Além disso, têm sido observadas nas empresas algumas formas de trabalho em grupo e rotação de tarefas, analisadas a seguir.

- Enriquecimento de cargos: Pode ocorrer por meio da ampliação horizontal, com a incorporação de tarefas de mesma natureza, da ampliação vertical, incorporação de tarefas de naturezas distintas, ou nos dois sentidos. O termo "célula de produção" é usado na empresa pesquisada para designar segmentos da linha de montagem nos quais há rodízio de trabalhadores na execução das operações. A intensão da rotação é que o trabalhador da produção seja capaz de operar máquinas de diferentes especialidades, localizadas em diferentes etapas do processo de produção ou atuar nas linhas de montagem dos distintos produtos da empresa. Não existia mais o preparador de máquinas e os operadores fazem manutenção de rotina. A rotação é outra forma de obter maior flexibilidade dos funcionários, que Salerno (1995) chama de 
flexibilidade dos trabalhadores quanto à função, relacionada à capacidade dos trabalhadores em desempenharem seu trabalho em postos de trabalho diferentes ou de serem realocados entre diferentes áreas de fabricação. Por levarem à ampliação de funções, os termos polivalência e multifuncionalidade têm sido usados para descrever essas mudanças. Segundo Smith (1997), a grande quantidade de cargos que existia antes, com especificação muito estreita de atividades para cada um, foi mudada para cargos com descrições que permitem a ampliação das diferentes atividades realizadas, diminuindo o número de classificações. Nesse contexto, é que se criaram os cargos de "operário multifuncional" (Rachid, 1996). Na empresa pesquisada, o cargo dos operários recém contratados é "operador de manufatura 1". Com as promoções, pode-se chegar até “operador de manufatura 6". Também neste caso, a descrição é aberta à incorporação de diferentes atividades.

Segundo Araújo et al. (2004), "um trabalhador com um conhecimento mais amplo da produção e capaz de realizar várias funções distintas passou a ser fundamental para acompanhar o movimento de flexibilização da [...] produção" (p.146). Para isso, as empresas têm investido em cursos e treinamentos. As Tabelas 1 e 2 apresentam, respectivamente, os tipos de treinamentos realizados nos últimos 12 meses e citados pelos funcionários entrevistados e o número de dias no total.

Tabela 1 - Tipos de cursos mencionados pelos trabalhadores $(\%)$

\begin{tabular}{|c|c|}
\hline Tipos de cursos e treinamentos mencionados & Porcentagem de entrevistados \\
\hline Meio ambiente, saúde e segurança no trabalho & 30,1 \\
\hline Gestão da qualidade & 29,7 \\
\hline Técnico-operacionais para a produção & 26,3 \\
\hline Gestão de recursos humanos & 11,8 \\
\hline Gestão da empresa & 1,3 \\
\hline Idiomas & 0,4 \\
\hline Gestão da produção & 0,4 \\
\hline
\end{tabular}

Uma porcentagem de $96,2 \%$ dos trabalhadores entrevistados tinha participado de algum treinamento nos doze meses anteriores à entrevista. Entre estes, 69,2\% tiveram até dois dias de treinamento. A empresa foi responsável pela oferta de $81,7 \%$ dos cursos mencionados. O gerente de recursos humanos disse que a empresa financiou supletivo de primeiro e segundo grau, curso de técnico de qualidade oferecido pelo SENAI e um grupo ligado a uma universidade local, o que foi mencionado por $9,4 \%$ e $2,6 \%$ dos trabalhadores, respectivamente. Eles mencionaram ainda $1,3 \%$ de cursos oferecidos por empresas de consultoria. 
Tabela 2 - Dias de treinamento dos trabalhadores nos 12 meses anteriores à pesquisa (\%)

\begin{tabular}{|c|c|}
\hline Número de dias & Porcentagem de entrevistados \\
\hline Nenhum & 1,9 \\
\hline Menos de um dia & 44,2 \\
\hline 1 a 2 dias & 23,1 \\
\hline 2 a 5 dias & 13,5 \\
\hline 5 a 10 dias & 7,7 \\
\hline 10 dias ou mais & 7,7 \\
\hline
\end{tabular}

A empresa tem ainda um "sistema de treinamento contínuo dentro da fábrica", voltado para a preparação do pessoal para trabalhar na fábrica e que envolve treinamentos elaborados para solução de problemas específicos.

Os treinamentos mais citados pelos operários foram aqueles voltados para meio ambiente, saúde e segurança no trabalho, logo seguidos pelos de gestão da qualidade. Houve 46 menções aos cursos da certificação pela ISO 14000 ou relacionado à questão ambiental. Outro curso muito citado, 39 vezes, foi o curso voltado para a certificação ISO 9000, sendo que houve mais 26 menções a cursos distintos sobre métodos relacionados à qualidade. Entre os cursos técnicooperacionais para a produção, destacaram-se as referências ao "sistema de treinamento contínuo dentro da fábrica", mencionado pelo gerente de recursos humanos, principalmente aqueles voltados para a montagem da lavadora.

Além dos cursos supletivo, a empresa exige pelo menos o segundo grau completo para contratação de operários. O Gráfico 2 apresenta o grau de escolaridade da amostra de entrevistados. Como pode ser observado, 92,4\% dos entrevistados tem escolaridade igual ou superior ao segundo grau completo.

Gráfico 2 - Escolaridade dos operários entrevistados na empresa pesquisada (2005)

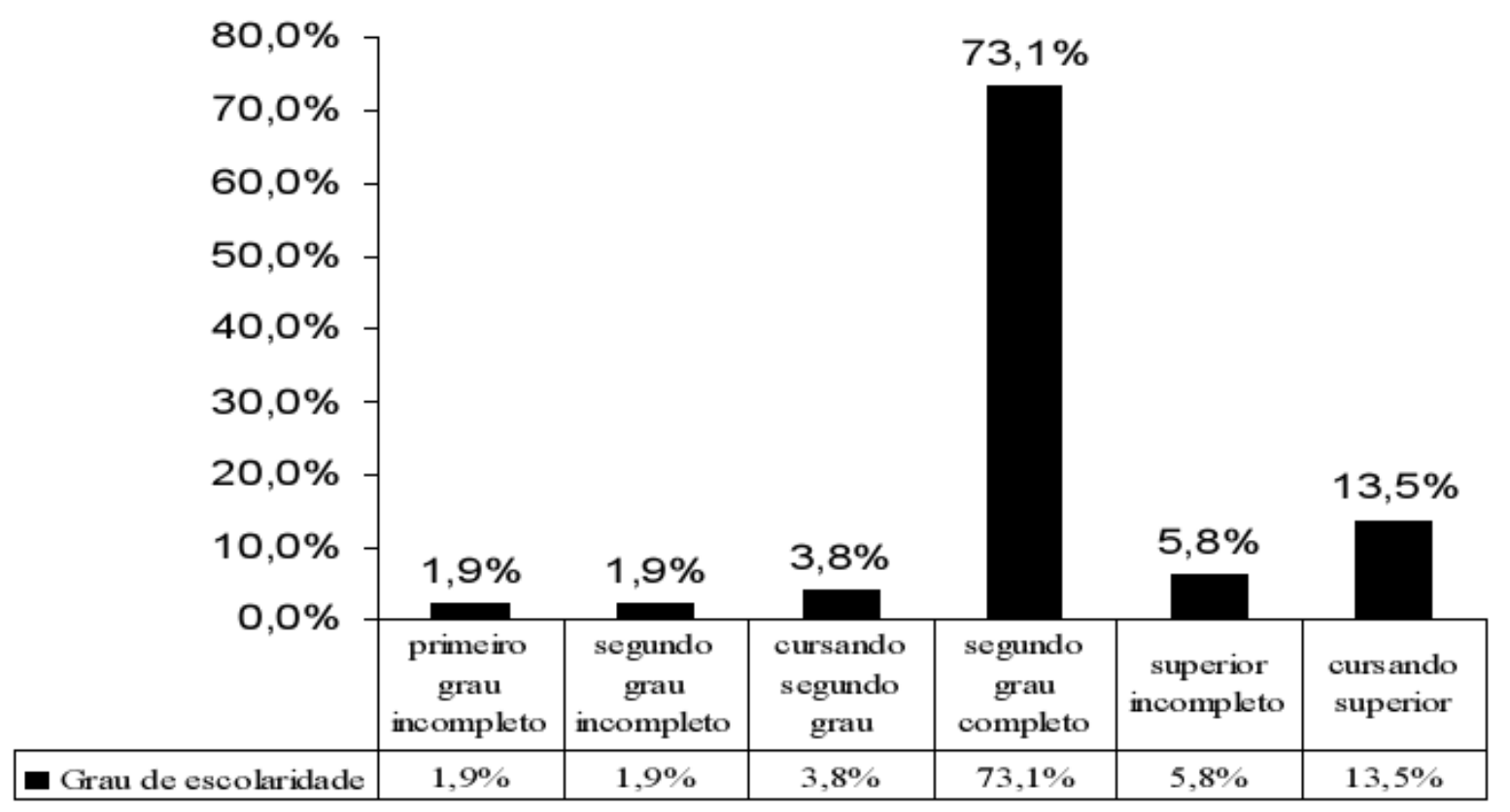


Contribuindo para o processo de racionalização, há o segundo tipo flexibilidade do trabalho, a numérica, quantitativa ou externa. Esta tem ocorrido juntamente com os processos de redução das estruturas organizacionais pelos quais têm passado as grandes empresas. Diversos autores têm apontado o aumento de formas distintas de contratação nos EUA e na Europa (Buonfiglio e Dowling, 2000; Felstead e Jewson, 1999; Fligstein e Shin, 2003; Hirata, 1998; Kalleberg, 2000, entre outros). Algumas formas alternativas de contratação, respondendo, de certa forma, à necessidade apontada por Noronha (2003), de identificar e discutir a "diversidade de tipos contratuais e suas formas de classificação" (p.112) são:

- Contrato temporário ou por prazo determinado, antes empregados para substituir pessoas em férias ou com licença e agora para preencher posições que costumavam ser permanentes (Smith, 1997);

- Terceirização, corte de áreas funcionais da estrutura organizacional das empresas levando à subcontratação de atividades de outras empresas para preencher esta lacuna (Smith, 1997);

- Remuneração variável, criação de uma parcela variável na composição dos salários, vinculada ao desempenho da empresa, programas de participação nos lucros e resultados, a PLR(Cacciamali, 2002; Martin, 1997);

- Trabalho em tempo parcial, antes uma estratégia individual para conciliar o trabalho com a vida pessoal e familiar, utilizada principalmente por mulheres com filhos pequenos ou por estudantes, hoje pessoas contratadas dessa forma por não encontrar outro tipo de emprego (Buonfiglio e Dowling, 2000; Felstead e Jewson, 1999; Fligstein e Shin, 2003; Kalleberg, 2000; Smith, 1997).

- Horas extra e banco de horas, comentados no próximo tópico.

\section{A flexibilidade da jornada de trabalho, análise da aplicação de acordos de banco de horas}

Devem ser numeradas em algarismos arábicos, apresentar título na parte superior e fonte na parte inferior, centralizado, fonte Times New Roman, tamanho 10, bem como ser referenciados no corpo do texto. Caso existam fotos ou figuras que demandem alta resolução, estas devem ser enviadas em arquivo separado em formato jpg e com a indicação da posição no texto.

A realização de hora extra e o banco de horas são feitos via um acordo entre o sindicato e a empresa, o que é posteriormente divulgado entre os operários e levado à votação conduzida pelo sindicato em assembléia. Na empresa pesquisada, esse acordo apresenta uma particularidade, pois parte das horas fora do expediente normal são pagas parte como horas extra e parte é anotada 
no banco de horas. O acordo é renovado a cada dois anos. Por este, fica acordado que as horas trabalhadas por iniciativa da empresa além da jornada regular de trabalho de 44 horas, são creditadas no banco de horas, sem que ocorra remuneração imediata pelo trabalho prestado, e as abaixo da jornada regular de trabalho, são debitadas do banco de horas, sem que ocorra prejuízo da remuneração correspondente. A jornada de trabalho não pode ultrapassar 10 horas diárias, não podendo ser computadas em sábados seguidos, feriados ou domingos.

Após o período de um ano, caso haja débito do funcionário, estas horas não trabalhadas são apenas anuladas, e, caso haja crédito, as horas a mais são pagas como horas extra. Também podem ser concedidas folgas adicionais, junto com o período de férias, ou ainda como parte das férias coletiva. Cabe ao funcionário comparecer às convocações da empresa nos dias e horário determinado. Caso não a cumpra, fica sujeito a desconto das horas no banco de horas. O mesmo ocorre em faltas não justificadas em dias de trabalho semanal. O adicional pago pela hora extra é de $50 \%$. O operário pode trabalhar uma hora adicional por dia via esta forma de pagamento e mais uma hora que é contabilizada no banco de horas. O banco abrange todos os operários, e para gerentes e supervisores a flexibilidade se dá de forma distinta, apesar de sua utilização não ser possível para todos.

Para gerentes e supervisores entrevistados, esta nova forma de contrato é primordial para a competitividade e manutenção de empregos no setor, já que existe uma sazonalidade na demanda que, caso não houvesse uma forma de flexibilização da jornada de trabalho, ocasionaria oscilação no quadro de funcionários, aumentando os níveis de rotatividade. O diretor do sindicato que representa os trabalhadores da empresa tem uma avaliação semelhante:

Como o mercado é sazonal, o banco permite que você, naquele momento em que a fábrica não está vendendo, fique em casa sem perder o seu salário. [...] Você ficou devendo essas horas para a empresa e vai pagar no dia em que a empresa estiver aquecida e necessitando.

Devem ser numeradas em algarismos arábicos, apresentar título na parte superior e fonte na parte inferior, centralizado, fonte Times New Roman, tamanho 10, bem como ser referenciados no corpo do texto. Caso existam fotos ou figuras que demandem alta resolução, estas devem ser enviadas em arquivo separado em formato jpg e com a indicação da posição no texto.

Entre os trabalhadores entrevistados, pode-se notar que há uma maior aceitação das horas extraordinárias como forma de flexibilizar a jornada de trabalho e que há divergência de opiniões a respeito da flexibilização da jornada de trabalho via banco de horas. As principais queixas a respeito deste tipo de contrato são quanto a:

- A obrigatoriedade de participação para os recém contratados, que além de não terem participado do processo de decisão quanto a adesão à este contrato, é obrigatória a 
adesão ao banco de horas no ato da contratação;

- A falta de regras que especifiquem mais detalhadamente as situações onde se pode utilizar o banco de horas, não sendo assim utilizados para citações cotidianas, como a falta material ou condições para o trabalho;

- O cansaço e fadiga que o banco de horas pode causar, já que a jornada de trabalho se desloca, se concentrado em determinadas épocas quando a produção requerida torna-se maior;

- A falta de credibilidade deste tipo de contrato quanto a seu objetivo de evitar demissões em épocas de depressões na produção, já que não existe legislação formal para evitar tais demissões pela empresa.

Entre os que concordam com o contrato, as principais vantagens apresentadas pelos operários foram quanto a:

- A estabilidade no emprego, evitando demissões em épocas de baixa demanda;

- Evitar perdas de direitos trabalhistas para trabalhadores que estão a mais tempo na empresa;

- A possibilidade de utilização das horas acumuladas no banco de horas para tratar de assuntos particulares quando estes são em horário de expediente;

- Possibilidade de utilização das horas acumuladas para estender os períodos de férias, para prolongar feriados, tanto individualmente como de forma coletiva.

\section{Considerações finais}

Devem ser numeradas em algarismos arábicos, apresentar título na parte superior e fonte na parte inferior, centralizado, fonte Times New Roman, tamanho 10, bem como ser referenciados no corpo do texto. Caso existam fotos ou figuras que demandem alta resolução, estas devem ser enviadas em arquivo separado em formato jpg e com a indicação da posição no texto.

A ampliação de funções dos operários, a chamada flexibilidade funcional, tem se dado principalmente pelo enriquecimento vertical, que é a incorporação de tarefas com natureza distinta, e horizontal, com a incorporação de atividades de mesma natureza, o que ocorrido principalmente devido ao rodízio entre os postos de trabalho.

As estratégias voltadas para a chamada flexibilidade numérica, por sua vez, não podem ser separadas da busca por maior racionalização no uso dos recursos. Na indústria de eletrodomésticos de linha branca, assim como em outras atividades consideradas como "metalúrgicas", tem havido a terceirização, o uso de contratos temporários ou por prazo 
determinado, a remuneração variável por meio da PLR (participação nos lucros e resultados), o uso de horas extra e do banco de horas.

O banco de horas, foco central de análise deste texto, é considerado como uma ferramenta que facilita muito a programação da produção, o que também tem sido apontado pelas pesquisas na área de PCP. No sindicato que representa os trabalhadores da empresa pesquisada, há uma avaliação de que este é uma necessidade das empresas e, que, por outro lado, auxilia na manutenção do emprego.

Entre trabalhadores, as opiniões se dividem. Parte dos entrevistados tem uma visão próxima da dos gerentes, de que é uma necessidade da programação da produção e que ajuda a manter os empregos. Alguns ressaltaram como o banco de horas abre possibilidades para se ausentar para cuidar de assuntos pessoais. Outros o vêem com restrições. A maior parte destes vê as horas extra como uma forma de aumentar o salário e o banco de horas acaba postergando ou restringindo o acesso a esse adicional. Ao contrário do que era esperado inicialmente, a partir de comentários de pesquisadores sobre o assunto, nenhum entrevistado mencionou que o banco de horas dificulta o planejamento de sua vida pessoal.

\begin{abstract}
This paper's objective is to analyze the strategies of labor flexibility in a white line home appliance enterprise, specially about annualized hours. The enterprises reordering since the nineties has been conducted to the flexibility. Considering specifically the labor flexibility, there are two different types that can be classified in: functional flexibility, which is the enlargement of the functions carried out by the workers, and numerical flexibility, that refers to the full time employment reduction and the increasing of alternatives forms of hiring, including annualized hours contracts. Interviews about the restructuring processes were taken with managers from the enterprise to gather information of these strategies. Taking into account the annualized hours contracts, the search involved 51 workers, human resources and factory managers, production overseers and a worker's unions director to knowing their viewpoint of this category of flexibility.
\end{abstract}

Key-words: work organization, work flexibility, flexible journey.

\title{
Referências
}

ALVES F.,A.G., RACHID, A., DONADONE, J. C., MARTINS, M. F., TRUZZI, O. S., BENTO, P. B., VANALLE, R. M. O Consórcio Modular e seus impactos na cadeia de suprimentos da fábrica de motores VW - São Carlos. Relatório final de projeto temático FAPESP (proc. 97/13071-9), 2001.

ARAÚJO, A.M.C., GITAHY, L., RACHID, A. e CUNHA, A. M. Globalização, estratégias gerenciais e respostas operárias: um estudo comparativo da indústria de linha branca. Relatório de projeto CNPq (proc. No. 403852/03-0), 2006.

ATKINSON, A. Changing working patterns. How companies achieve flexibility to meet new needs. Londres, NEDO (National Economic Development Office), 1986.

BUONFIGLIO, M.C. \& DOWLING, J.A. Flexibilidade das relações de trabalho e precarização uma análise 
comparativa. In: XXIV Encontro Anual da ANPOCS, Caxambu, 2000.

CACCIAMALI, M.C. Princípios e direitos fundamentais no trabalho na América Latina. In: São Paulo em Perspectiva, Apr/Jun, vol.16, n.2. p.64-75, 2002.

DAL ROSSO, S. Flextempo - flexibilização da jornada à brasileira. In: M.C. Ferreira e S. Dal Rosso (org.) A regulação social do trabalho. Brasília, Ed. Paralelo 15. p. 71-92, 2003.

FELSTEAD, A. \& JEWSON, N. Flexible labour and non-standard employment: na agenda of issues. In: (ed.) Global trends in flexible labour, Macmillan, p.1-20, 1999.

FLIGSTEIN, N. \& SHIN, T. The shareholder value society: a review of the changes in working conditions and inequality in the U.S. 1976-2000. Working Paper, University of Califórnia, Berkeley. 64p. Disponível no site sociology.berkeley.edu/faculty/ fligstein, 2003.

GADREY, J. Emprego, produtividade e avaliação do desempenho dos serviços. In: Seminário Os estudos do trabalho: novas problemáticas, novas metodologias e novas áreas de pesquisa. São Paulo, CEBRAP/ USP/ UNICAMP, dezembro. 36p., 1999.

GERWIN, D. An agenda for research on the flexibility of manufacturing processes. In: International Journal of Operations \& Production Management, vol. 7, n. 1, p.38-49. Republicado no vol. 25, n. 12, 2005, pp. 1171-1182, 1987.

HIRATA, H. Entre mercado de trabalho, organização da produção e resistência dos trabalhadores: os meandros atuais da flexibilidade. In: Seminário Internacional "Reestruturação produtiva, flexibilidade do trabalho e novas competências profissionais", COPPE/UFRJ, Rio de Janeiro, agosto, 1998.

INVERNIZZI, N. Novos rumos do trabalho. Mudanças na forma de controle e qualificação da força de trabalho brasileira. DPCT/UNICAMP, Campinas, 2000. (Teses de doutorado), 2000.

KALLEBERG, A.L. Nonstandard employment relations - part-time, temp and contract work. In: Annual Review of Sociology, n.26. p.341-365, 2000.

cross ${ }^{\text {ref }}$

MARTÍNEZ, M. A construção da qualificação: um estudo de caso na indústria de linha branca. Dissertação de mestrado apresentada ao Programa de pós - graduação em Engenharia de Produção da Universidade Federal de São Carlos para obtenção do título de Mestre em Engenharia de Produção, São Carlos, SP, 2004.

RACHID, A. O Brasil Imita o Japão? A Qualidade em empresas de Autopeças. In: Cadernos de Gestão Tecnológica, n.32, São Paulo, CYTED: NPGCT/USP. 67p. Disponível em www.fea.usp.br/programas/cyted/ subprogramaXVI/cadernos.htm,1996.

MARX, R. Trabalho em grupos e autonomia como instrumentos da competição. São Paulo, Ed. Atlas, 1997.

ROTTA, I. S. Mini-fábrica: Uma nova proposta de arranjo produtivo e organizacional híbrido em uma empresa do setor eletrônico. Tese apresentada ao Programa de pós - graduação em Engenharia de Produção da Universidade Federal de São Carlos para obtenção do título de Doutor em Engenharia de Produção, São Carlos, SP, 2004.

SALERNO, M.S. Flexibilidade e organização produtiva. In: Nadya A. Castro (org.) A máquina e o equilibrista: inovações na indústria automobilística brasileira. São Paulo, Paz e Terra. p.53-83, 1995.

SMITH, V. New forms of work organization. In: Annual Review of Sociology, v.23. p.315-339. Disponível no site www.periodicos.capes.gov.br, 1997.

WALBY, S. As figuras emblemáticas do emprego flexível. In: Margaret Maruani e Helena Hirata (org.) As novas fronteiras da desigualdade, São Paulo, Ed. SENAC. p.287-298, 2003.

WOOD, S. The transformation of work? In:

labour process. Boston, Unwin Hyman. p.1-43, 1987.

(org.) The transformation of work? Skill, flexibility and the 


\section{Dados dos autores}

Autor 1

Nome completo: Lílian Carneiro Souza

Filiação institucional: Universidade Federal de São Carlos

Departamento: Departamento de engenharia de produção

Função ou cargo ocupado: ex-graduanda

Endereço completo para correspondência (bairro, cidade, estado, país e CEP): Avenida das Gardênias, $\mathrm{n}^{\circ}$ 241, apto. 12, bairro Cidade Jardim, São Carlos, São Paulo, Brasil. CEP: 13566-540

Telefones para contato: (16) 9186-8424

e-mail: 1liancarneirosouza@yahoo.com.br

Autor 2

Nome completo: Alessandra Rachid

Filiação institucional: Universidade Federal de São Carlos

Departamento: Departamento de engenharia de produção

Função ou cargo ocupado: Professora adjunto

Endereço completo para correspondência (bairro, cidade, estado, país e CEP): DEP-UFSCar - Rod.

Washington Luis, km 235 - Caixa Postal 676 - CEP 13565-905 - São Carlos - SP

Telefones para contato: 16-3351-8236

e-mail: arachid@power.ufscar.br

Recebido para publicação em: 04/02/2008

Aceito para publicação em: 06/03/2008 